\title{
Enhancing phase sensitivity with number state filtered coherent states
}

\author{
Nilakantha Meher $^{a}$. S. Sivakumar ${ }^{b}$
}

Received: date / Accepted: date

\begin{abstract}
Number state filtered coherent states are a class of nonclassical states obtained by removing one or more number states from a coherent state. Phase sensitivity of an interferometer is enhanced if these nonclassical states are used as input states. The optimal phase sensitivity, which is related to the quantum Cramer-Rao bound (QCRB) for the input state, improves beyond the standard quantum limit. It is argued that removal of more than one suitable number state leads to better phase sensitivity. As an important limiting case in this context, the even and odd coherent states, where the odd and even number states are filtered from coherent state respectively, are considered. The optimal phase sensitivity for these limiting cases equals that of the squeezed vacuum. It is observed that the improvement in phase sensitivity is not in direct proportion to the nonclassicality of the input states.
\end{abstract}

Keywords Number state filtering · Quantum Fisher information · Quantum metrology

\section{Introduction}

Interferometery is the primary tool for estimating change of phase of optical fields $1,13,19,23]$. It relies on the changes in the interference pattern when a phase object is introduced in one of the arms of an interferometer, for instance, the Mach-Zehnder interferometer (MZI). An important requirement in phase measurement is to achieve precision as close to the theoretical bound given by the laws of quantum mechanics, which are much lower than the classical limit on interferometric phase measurements. Phase sensitivity of an interferometric configuration depends on the states of the light at the two input ports.

Among the pure states of light, the coherent states are important as they possess features such as factorizable coherence functions, localized phase space distribution, minimal quadrature fluctuations, etc [21, 22, 41]. Aptly, these states are classified as the most classical among the quantum states. With the classical states, i.e., coherent state, as one of the input states and vacuum as the other input, phase sensitivity is limited by the standard quantum limit (SQL), i.e., $\Delta \theta_{\mathrm{SQL}}=1 / \sqrt{N}$, where $N$ is the average number of photons in the coherent state [13, 17]. In this arrangement, sensitivity can be enhanced by increasing the average number of photons. But higher intensity of the field increases the radiation pressure which decreases sensitivity [10, 11]. However, SQL is not the lowest bound for

\footnotetext{
${ }^{a}$ Department of Physics, Indian Institute of Technology Kanpur, Kanpur, UP 208016, India.

${ }^{b}$ Division of Natural Sciences, Krea University, Andhra Pradesh 517646, India,

E-mail: ${ }^{a}$ nilakantha.meher6@gmail.com · E-mail: ${ }^{b}$ sivakumar.srinivasan@krea.edu.in
} 
phase measurements. If suitable nonclassical states are used as input states sensitivity can be improved [16, 20]. For instance, squeezed vacuum (SV) and coherent state in the input of MZI beats the standard quantum limit [11]. The optimal phase shift that can be resolved using these input states is $\Delta \theta=e^{-r} / \sqrt{N}$, where $r$ is the squeezing parameter 32 . The minimum phase shift that can be detected in an interferometer is bounded by the Heisenberg limit (HL) [10, 17], i.e. $\Delta \theta_{\mathrm{HL}}=1 / N$, where $N$ is the average number of photons input to the interferometer. Enhancement of phase sensitivity beyond SQL can be achieved using nonclassical states in the input. For instance, NOON state [6, 24, 27], entangled coherent state 28, 29, 34], pair coherent state [18], twin Fock state 9, 33], squeezed thermal state and even or odd coherent states [42], coherent state and number state [4], two mode squeezed states [5], etc improve phase sensitivity of interferometer.

Another interesting case is to replace the vacuum port with a coherent state input so that the input is a double coherent state, i.e., $|\alpha\rangle|\beta\rangle$. The optimal phase sensitivity that can be achieved is $\Delta \theta=1 / \sqrt{|\alpha|^{2}+|\beta|^{2}}$ [2]. If $\alpha=\beta$, then $\Delta \theta=1 / \sqrt{2 N}, N$ being the average number of photons in any one of the inputs. Substituting the vacuum with a coherent state in the input improves the phase sensitivity. However, it does not go below SQL. This arises from the fact that the classical input states remain classical at the output of MZI.

It is of interest to construct input states that overcome SQL. In this article, we investigate the phase sensitivity of a special class of non-classical states called the number state filtered coherent states (NFCS) 37]. Quantum Cramer-Rao bound (QCRB) gives the optimal phase sensitivity achievable for a given input state [12, 31, 40]. Hence, QCRB is used to quantify the achievable phase sensitivity. Using NFCS and coherent state, Heisenberg limit is asymptotically reached for large average photon number. Filtering a single number state from coherent state improves the phase sensitivity. It can be improved further by filtering more than one number state from the coherent state. We show that the odd coherent state (OCS) and even coherent state (ECS), where even and odd photon number states are filtered out, perform as well as SV for phase measurement.

This article is organized as follows: A brief review of calculation of quantum Fisher information (QFI) and QCRB is given in Sec. 2. Phase sensitivity of single number state filtered coherent state is discussed in Sec. 3. In Sec. 4, the optimal phase sensitivity achievable using multi-number states filtered coherent state such as ECS and OCS is presented. We discuss the role of nonclassicality of the quantum state on phase sensitivity in Sec. 5. Finally, we summarize the results in Sec. 6]

\section{Quantum Fisher information}

In this section, a brief overview on quantum Fisher information of the input state to the MZI is presented. Interestingly, QFI is related to the quantum Cramer-Rao bound [25, 26, 31, 33], which provides a lower bound in phase sensitivity. Recent studies have employed QFI in various contexts such as an hierarchical ordering of quantum states [15], characterizing the non-Markovianity of open quantum processes [35], understanding the thermal entanglement [39], etc. This measure has also been investigated in studying the robustness of W-states [38] and GHZ states 36] under dissipation and decoherence. For optimizing the phase sensitivity of an interferometer in the presence of dissipation or decoherence, Heisenberg-Langevin approach or quantum master equation approach can be implemented to calculate QFI [43]. However, in this work we have assumed that dissipation and decoherence are negligible and are neglected.

Let the input state to the interferometer be a product state of the form $\left|\psi_{\text {in }}\right\rangle=\left|\psi_{1}\right\rangle\left|\psi_{2}\right\rangle$, where $\left|\psi_{1}\right\rangle$ and $\left|\psi_{2}\right\rangle$ are the input states to the ports 1 and 2 respectively as indicated in Fig. 1] Relevant 


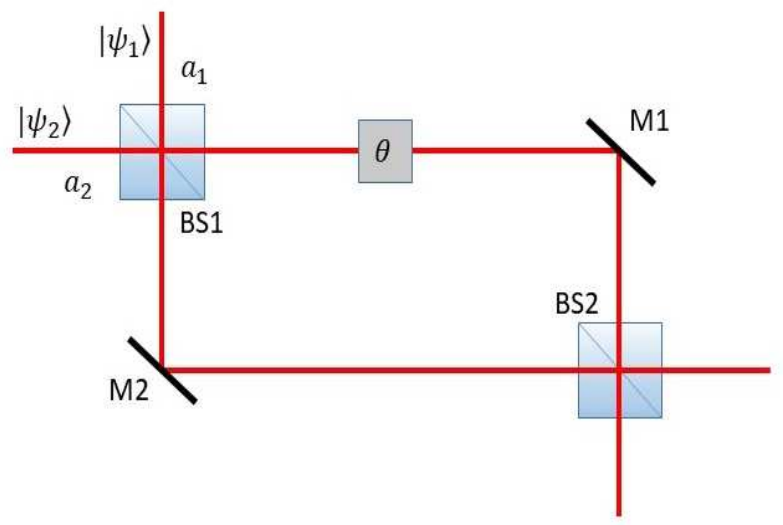

Fig. 1 Sketch of a Mach-Zhender interferometer. Two states $\left|\psi_{1}\right\rangle$ and $\left|\psi_{2}\right\rangle$ are the input to the ports 1 and 2 respectively. The phase element in the upper arm introduces a relative phase $\theta$ between the paths.

to phase sensitivity of MZI is the QFI of the state that results after the action of the first beam splitter and phase shift due to the phase object. The unitary operator to represent the action of the first beam splitter $(50: 50)$ is $U_{B S}=e^{-i \frac{\pi}{4}\left(a_{1}^{\dagger} a_{2}+a_{1} a_{2}^{\dagger}\right)}$ and for the relative phase shift the operator is $U(\theta)=e^{-i \theta\left(a_{1}^{\dagger} a_{1}-a_{2}^{\dagger} a_{2}\right) / 2}$.

Defining

$$
\begin{gathered}
J_{x}=\frac{1}{2}\left(a_{1}^{\dagger} a_{2}+a_{1} a_{2}^{\dagger}\right), \\
J_{y}=\frac{-i}{2}\left(a_{1}^{\dagger} a_{2}-a_{1} a_{2}^{\dagger}\right), \\
J_{z}=\frac{1}{2}\left(a_{1}^{\dagger} a_{1}-a_{2}^{\dagger} a_{2}\right),
\end{gathered}
$$

The unitary operators are recast as $U_{B S}=\exp \left(\frac{-i J_{x} \pi}{2}\right)$ and $U_{\theta}=\exp \left(-i \theta J_{z}\right)$ respectively.

Quantum Fisher information for a pure state $\left|\psi_{\text {in }}\right\rangle$ is 7, 26, 31, 33.

$$
F_{Q}(\theta)=4\left\{\left\langle\psi^{\prime}(\theta) \mid \psi^{\prime}(\theta)\right\rangle-\left|\left\langle\psi^{\prime}(\theta) \mid \psi(\theta)\right\rangle\right|^{2}\right\},
$$

where

$$
|\psi(\theta)\rangle=U(\theta) U_{B S}\left|\psi_{i n}\right\rangle=e^{-i \theta J_{z}} \exp \left(-\frac{i J_{x} \pi}{2}\right)\left|\psi_{i n}\right\rangle
$$

and

$$
\left|\psi^{\prime}(\theta)\right\rangle=\frac{\partial}{\partial \theta}|\psi(\theta)\rangle=-i e^{-i \theta J_{z}} J_{z} \exp \left(-\frac{i J_{x} \pi}{2}\right)\left|\psi_{i n}\right\rangle .
$$

Using the Eqns. 5 and 6, QFI given in Eqn. 4 becomes

$$
F_{Q}(\theta)=4\left[\left\langle\psi_{i n}\left|J_{y}^{2}\right| \psi_{i n}\right\rangle-\left\langle\psi_{i n}\left|J_{y}\right| \psi_{i n}\right\rangle^{2}\right],
$$

which is proportional to the variance of $J_{y}$ in the input state $\left|\psi_{i n}\right\rangle$. Here we have used the identity

$$
\exp \left(\frac{i J_{x} \pi}{2}\right) J_{z} \exp \left(-\frac{i J_{x} \pi}{2}\right)=J_{y}
$$


Using $J_{y}=\frac{-i}{2}\left(a_{1}^{\dagger} a_{2}-a_{1} a_{2}^{\dagger}\right)$, Eqn. 7 can be written as

$$
\begin{aligned}
& F_{Q}(\theta)=\left\langle a_{1}^{\dagger} a_{1}\right\rangle+\left\langle a_{2}^{\dagger} a_{2}\right\rangle-\left\langle a_{1}^{\dagger 2} a_{2}^{2}\right\rangle-\left\langle a_{1}^{2} a_{2}^{\dagger 2}\right\rangle \\
& \quad+2\left\langle a_{1}^{\dagger} a_{1} a_{2}^{\dagger} a_{2}\right\rangle+\left\langle a_{1}^{\dagger} a_{2}\right\rangle^{2}+\left\langle a_{1} a_{2}^{\dagger}\right\rangle^{2}-2\left\langle a_{1}^{\dagger} a_{2}\right\rangle\left\langle a_{1} a_{2}^{\dagger}\right\rangle,
\end{aligned}
$$

where \langle\rangle implies expectation value in the input state $|\psi\rangle_{i n}$.

The phase shift that can be resolved by a given input state in an interferometric arrangement is bounded by $17,11,26,31,33$

$$
\Delta \theta \geq \frac{1}{\sqrt{F_{Q}(\theta)}},
$$

the quantum Cramer-Rao bound [12, 40]. The optimal phase shift $\Delta \theta_{Q C R B}=1 / \sqrt{F_{Q}(\theta)}$. This relationship implies that for better phase sensitivity, the states input to the MZI should have larger $F_{Q}(\theta)$.

We assume that the input to the port 2 is a coherent state $|\beta\rangle$ throughout the discussion. For this choice,

$$
F_{Q}(\theta)=\left\langle a_{1}^{\dagger} a_{1}\right\rangle+|\beta|^{2}+2|\beta|^{2}\left(\left\langle a_{1}^{\dagger} a_{1}\right\rangle-\left\langle a_{1}^{\dagger}\right\rangle\left\langle a_{1}\right\rangle\right)-|\beta|^{2}\left(\left\langle a_{1}^{\dagger 2}\right\rangle+\left\langle a_{1}^{2}\right\rangle-\left\langle a_{1}^{\dagger}\right\rangle^{2}-\left\langle a_{1}\right\rangle^{2}\right) .
$$

The expectation values of operators for the input port 1 appearing in Eqn. 11 are to be calculated in $\left|\psi_{1}\right\rangle$.

\section{Single number state filtered coherent state}

In this section, we study the phase sensitivity of single number state filtered coherent state (SNFCS) in terms of quantum Cramer-Rao bound. SNFCS is defined as [37]

$$
|\psi(\alpha, m)\rangle=\frac{e^{-|\alpha|^{2} / 2}}{N_{m}} \sum_{n=0, n \neq m}^{\infty} \frac{\alpha^{n}}{\sqrt{n !}}|n\rangle,
$$

where $N_{m}=\sqrt{1-e^{-|\alpha|^{2}} \frac{|\alpha|^{2 m}}{m !}}$. This definition implies that the number state $|m\rangle$ is absent from the coherent state. Hence, the probability of detecting $m$ photons in $|\psi(\alpha, m)\rangle$ is zero.

3.1 Phase sensitivity with SNFCS and coherent state input

In order to investigate the advantages of using SNFCS, consider the input state $\left|\psi_{i n}\right\rangle=|\psi(\alpha, m)\rangle|\beta\rangle$, wherein the vacuum state in the input port has been replaced with $|\psi(\alpha, m)\rangle$. From Eqn. 11] QFI associated with this input is

$$
F_{\mathrm{S}}(\theta)=\left\langle a_{1}^{\dagger} a_{1}\right\rangle+|\beta|^{2}+2|\beta|^{2}\left(\left\langle a_{1}^{\dagger} a_{1}\right\rangle-\left\langle a_{1}^{\dagger}\right\rangle\left\langle a_{1}\right\rangle\right)-|\beta|^{2}\left(\left\langle a_{1}^{\dagger}\right\rangle+\left\langle a_{1}^{2}\right\rangle-\left\langle a_{1}^{\dagger}\right\rangle^{2}-\left\langle a_{1}\right\rangle^{2}\right),
$$

where

$$
\begin{aligned}
\left\langle a_{1}^{\dagger} a_{1}\right\rangle & =\frac{1}{N_{m}^{2}}\left[|\alpha|^{2}-m\left(1-N_{m}^{2}\right)\right], \\
\left\langle a_{1}\right\rangle & =\alpha+\frac{m}{\alpha^{*}}-\frac{m}{\alpha^{*} N_{m}^{2}}, \\
\left\langle a_{1}^{2}\right\rangle & =\alpha^{2}+\frac{m(m-1)}{\alpha^{* 2}}-\frac{m(m-1)}{\alpha^{* 2} N_{m}^{2}} .
\end{aligned}
$$



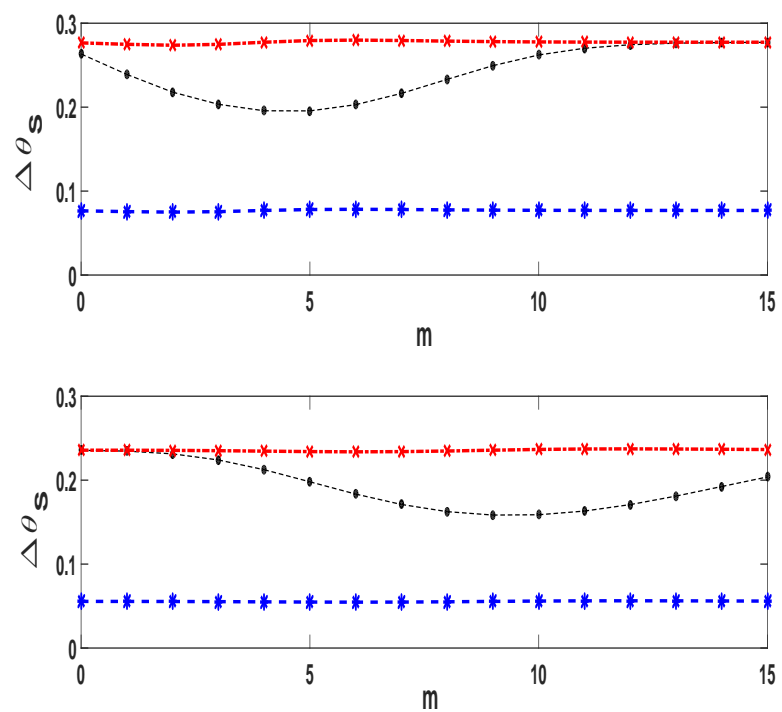

Fig. 2 Quantum Cramer-Rao bound $\Delta \theta_{\mathrm{S}}$ (larger dot) for the input state $\left|\psi_{\text {in }}\right\rangle=|\psi(\alpha, m)\rangle|\beta\rangle$ as a function of $m$. Here $(a) \alpha=2, \beta=3$ and $(b) \alpha=3, \beta=3$. It is compared with $\Delta \theta_{\mathrm{HL}}$ (star) and $\Delta \theta_{\mathrm{SQL}}$ (cross). Phase sensitivity beats standard quantum limit

The expectation values of operators relevant for the input port 1 are calculated in $|\psi(\alpha, m)\rangle$.

QCRB for the aforementioned input state is

$$
\Delta \theta_{\mathrm{S}}=\frac{1}{\sqrt{F_{\mathrm{S}}(\theta)}}
$$

and the corresponding HL and SQL are

$$
\Delta \theta_{\mathrm{HL}}=\frac{1}{\frac{1}{N_{m}^{2}}\left[|\alpha|^{2}-m\left(1-N_{m}^{2}\right)\right]+|\beta|^{2}}
$$

and

$$
\Delta \theta_{\mathrm{SQL}}=\frac{1}{\sqrt{\frac{1}{N_{m}^{2}}\left[|\alpha|^{2}-m\left(1-N_{m}^{2}\right)\right]+|\beta|^{2}}} .
$$

In Fig. 2. $\Delta \theta_{\mathrm{S}}$ is shown as a function of $m$. For comparison, $\Delta \theta_{\mathrm{HL}}$ and $\Delta \theta_{\mathrm{SQL}}$ are also shown. It is to be noted that the optimal phase sensitivity $\Delta \theta_{\mathrm{S}}$ surpasses SQL if SNFCS is used instead of vacuum state as the input in MZI. This indicates that filtering a number state from coherent state enhances phase sensitivity in an interferometric phase measurement. Moreover, maximum sensitivity is achieved when $|\alpha|^{2}=m$, as seen in Fig. 2. On the other hand, if $|\alpha|^{2}>>m$ or $|\alpha|^{2}<<m, \Delta \theta_{\mathrm{S}}$ reaches $\Delta \theta_{\mathrm{SQL}}$. In this limit $|\langle\psi(\alpha, m) \mid \alpha\rangle|^{2} \approx 1$, which implies $|\psi(\alpha, m)\rangle \approx|\alpha\rangle[37$.

Interestingly, for $|\alpha|^{2}=|\beta|^{2}>>1$ and $m=|\alpha|^{2}, \Delta \theta_{\mathrm{S}}$ given in Eqn. 17 becomes

$$
\Delta \theta_{\mathrm{S}}=\frac{\sqrt{2}}{N} \sqrt{\frac{N_{m}^{2}}{1-N_{m}^{2}}},
$$




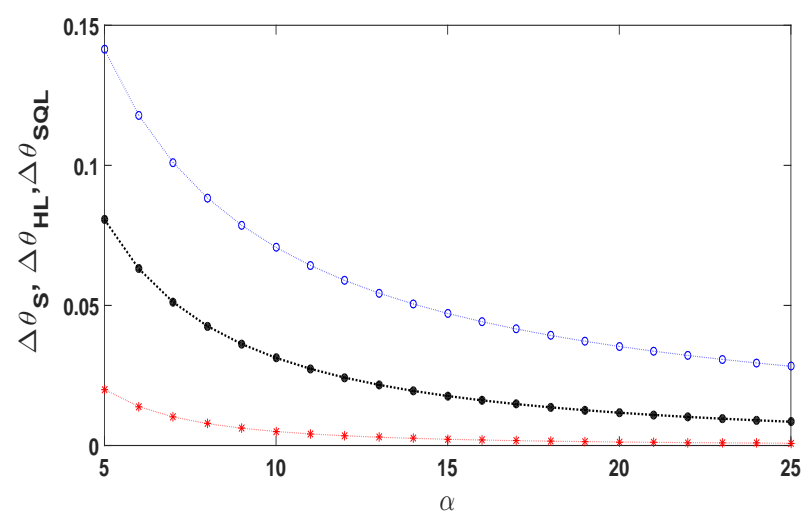

Fig. 3 Quantum Cramer-Rao bound $\Delta \theta_{\mathrm{S}}$ for the input state $\left|\psi_{i n}\right\rangle=|\psi(\alpha, m)\rangle|\beta\rangle$ (middle curve). Result is compared with Heisenberg limit $\Delta \theta_{\mathrm{HL}}$ (lower curve) and SQL $\Delta \theta_{\mathrm{SQL}}$ (upper curve). Here we assume $\alpha=\beta$, and $m=|\alpha|^{2}, \alpha$ and $\beta$ are assumed as real.

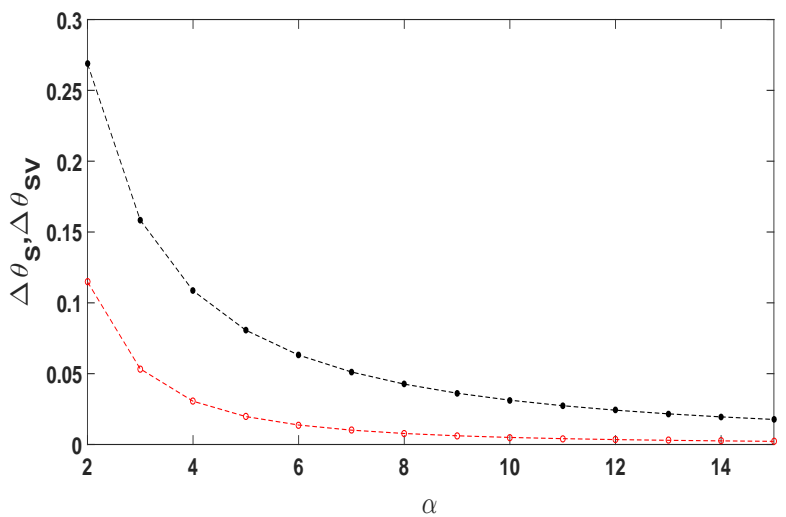

Fig. $4 \Delta \theta_{\mathrm{S}}$ and $\Delta \theta_{\mathrm{SV}}$ are shown as a function of $\alpha$ respectively for the input states $|\psi(\alpha, m)\rangle|\beta\rangle$ (upper curve) and $|\xi\rangle|\beta\rangle$ (lower curve). Here we assume $\alpha=\beta, m=|\alpha|^{2}$ and $r=\sqrt{\sinh ^{-1}|\alpha|^{2}}$. $\alpha$ and $\beta$ are assumed as real.

which shows the Heisenberg scaling $\Delta \theta_{\mathrm{S}} \propto 1 / N$, as can be seen in Fig. 3. Here $N=|\alpha|^{2}+|\beta|^{2}$, which is the total average number of photons at the MZI input. It is to be noted that the average number of photons in $|\psi(\alpha, m)\rangle$ is $|\alpha|^{2}$ when $m=|\alpha|^{2}$.

It is known that SV and coherent state at the input of a MZI achieves optimal phase sensitivity. As a comparison, we show QCRB for $|\psi(\alpha, m)\rangle|\beta\rangle$ and $|\xi\rangle|\beta\rangle$ in Fig. 4, where $|\xi\rangle$ is SV. For the input state $|\xi\rangle|\beta\rangle, \mathrm{QCRB}$ is 10,11$]$

$$
\Delta \theta_{\mathrm{SV}}=\frac{1}{\sqrt{|\beta|^{2} e^{2 r}+\sinh ^{2} r}}
$$

where $r$ is the squeezing parameter chosen in such a way that $|\alpha|^{2}=\sinh ^{2} r$. This condition ensures that the average number of photons in $|\xi\rangle$ and $|\psi(\alpha, m)\rangle$ are equal if $m=|\alpha|^{2}$. As can be seen in Fig. 4. $\Delta \theta_{\mathrm{SV}}<\Delta \theta_{\mathrm{S}}$, which indicates that the performance of SV is better than that of SNFCS. 


\section{Multi-number states filtered coherent state}

If more than one number state are filtered from a coherent state, the resultant state is a multi-number states filtered coherent state (MNFCS). In the number state basis

$$
|\psi(\alpha,\{m\})\rangle \propto e^{-|\alpha|^{2} / 2} \sum_{n=0, n \notin\{m\}}^{\infty} \frac{\alpha^{n}}{\sqrt{n !}}|n\rangle,
$$

where the set $\{m\}$ contains more than one integer. If $\{m\}$ has only a single member, this state reduces to the state given in Eqn. 12 which is the SNFCS.

Two special classes of states, namely, even coherent state and odd coherent state belong to this category [8, 14]. If the set $\{m\}$ contains only odd (even) integers, then the resultant state is even (odd) coherent state. Number state representation of ECS and OCS are

$$
|\alpha,+\rangle \propto e^{-|\alpha|^{2} / 2} \sum_{n=0}^{\infty} \frac{\alpha^{2 n}}{\sqrt{(2 n) !}}|2 n\rangle,
$$

and

$$
|\alpha,-\rangle \propto e^{-|\alpha|^{2} / 2} \sum_{n=0}^{\infty} \frac{\alpha^{(2 n+1)}}{\sqrt{(2 n+1) !}}|2 n+1\rangle,
$$

respectively. These states are nonclassical [8, 14].

\subsection{Phase sensitivity using MNFCS and coherent state}

Let the input state to MZI is $|\psi(\alpha,\{m\})\rangle|\beta\rangle$. QCRB for this input can be calculated using the Eqn. 11 where the expectation values are to be calculated in the state $|\psi(\alpha,\{m\})\rangle$. We denote the QCRB for this input as $\Delta \theta_{\mathrm{M}}$.

Define

$$
\Theta=\frac{\Delta \theta_{\mathrm{M}}}{\Delta \theta_{\mathrm{HL}}}
$$

to quantify the effectiveness of the input state. The case $\Theta=1$ corresponds to the ultimate phase sensitivity achievable. Here $\Delta \theta_{\mathrm{HL}}=1 /\left[\left\langle a_{1}^{\dagger} a_{1}\right\rangle+|\beta|^{2}\right]$, where $\left\langle a_{1}^{\dagger} a_{1}\right\rangle=\left\langle\psi(\alpha,\{m\})\left|a_{1}^{\dagger} a_{1}\right| \psi(\alpha,\{m\})\right\rangle$ is the average number of photon in MNFCS .

The ratio $\Theta$ depends on the choice of $\{m\}$. The optimal choice is the set which yields the lowest $\Delta \theta_{M}$ for a given $\alpha$ and $\beta$. The ratio $\Theta$ for the input $|\psi(\alpha,\{m\})\rangle|\beta\rangle$ is shown in Fig. 5 as a function of $k$. Here $k$ denotes the number of Fock state filtered, equivalently, number of elements in the set $\{m\}$. For example, SNFCS corresponds to $k=1$ as the set $\{m\}$ contains only one integer. It is to be noted that as $k$ increases, i.e., number of Fock state filtered from the coherent state increases, $\Theta$ decreases. This signifies an improvement of phase sensitivity. Hence, filtering number states from coherent state enhances phase sensitivity.

QCRB for $|\alpha,+\rangle|\beta\rangle$ as the input state is

$$
\Delta \theta_{+}=\frac{1}{\sqrt{\left\langle a_{1}^{\dagger} a_{1}\right\rangle+|\beta|^{2}\left(1+2\left\langle a_{1}^{\dagger} a_{1}\right\rangle-2|\alpha|^{2} \cos 2 \theta\right)}},
$$




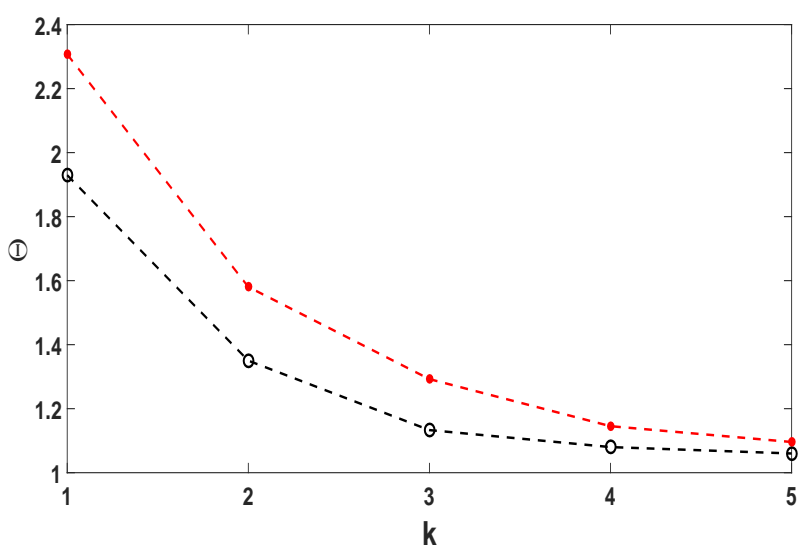

Fig. $5 \Theta$ is shown as a function of number of elements $(k)$ in the set $\{m\}$ for the state $|\psi(\alpha,\{m\})\rangle|\beta\rangle$ where $\alpha=3 e^{i \pi / 2}$ (circle) and $4 e^{i \pi / 2}(\operatorname{dot})$. We set $\beta=|\alpha|$.

where $\theta=\arg (\alpha)$ and

$$
\left\langle a_{1}^{\dagger} a_{1}\right\rangle=\left\langle\alpha,+\left|a_{1}^{\dagger} a_{1}\right| \alpha,+\right\rangle=|\alpha|^{2} \tanh |\alpha|^{2},
$$

is the average number of photons in ECS.

A similar expression for QCRB for the input state $|\alpha,-\rangle|\beta\rangle$, i.e., $\Delta \theta_{-}$is given by substituting $\left\langle\alpha,+\left|a_{1}^{\dagger} a_{1}\right| \alpha,+\right\rangle$ by $\left\langle\alpha,-\left|a_{1}^{\dagger} a_{1}\right| \alpha,-\right\rangle$ in Eqn. [26, Here

$$
\left\langle\alpha,-\left|a_{1}^{\dagger} a_{1}\right| \alpha,-\right\rangle=|\alpha|^{2} \operatorname{coth}|\alpha|^{2}
$$

is the average number of photons in OCS. For $|\alpha|>>1,\left\langle\alpha,+\left|a_{1}^{\dagger} a_{1}\right| \alpha,+\right\rangle \approx\left\langle\alpha,-\left|a_{1}^{\dagger} a_{1}\right| \alpha,-\right\rangle \approx|\alpha|^{2}$.

Fig. 6] shows $\Delta \theta_{S V}, \Delta \theta_{-}$and $\Delta \theta_{+}$as a function of $|\alpha|$ for $\theta=\pi / 2$. We choose $\alpha$ and $r$ such that $\left\langle\alpha,+\left|a_{1}^{\dagger} a_{1}\right| \alpha,+\right\rangle \approx\left\langle\alpha,-\left|a_{1}^{\dagger} a_{1}\right| \alpha,-\right\rangle \approx\left\langle\xi\left|a_{1}^{\dagger} a_{1}\right| \xi\right\rangle$. All the three curves coincide for large average photon number. For $|\alpha|^{2}>>1$,

$$
\Delta \theta_{+} \approx \Delta \theta_{-} \approx \frac{1}{\sqrt{|\alpha|^{2}+|\beta|^{2}+4|\alpha|^{2}|\beta|^{2}}} \approx \frac{1}{\sqrt{|\beta|^{2} e^{2 r}+\sinh ^{2} r}}
$$

which are equal to the $\Delta \theta_{\mathrm{SV}}$ given in Eqn. 21] Hence, ECS and OCS perform as good as SV for phase measurement.

\section{Nonclassicality and phase sensitivity}

Phase sensitivity of an interferometric setup using classical inputs can not surpass standard quantum limit. It requires one of the inputs to be non-classical to go beyond SQL. A pertinent question that arises is whether a maximally nonclassical state shows maximum phase sensitivity. 


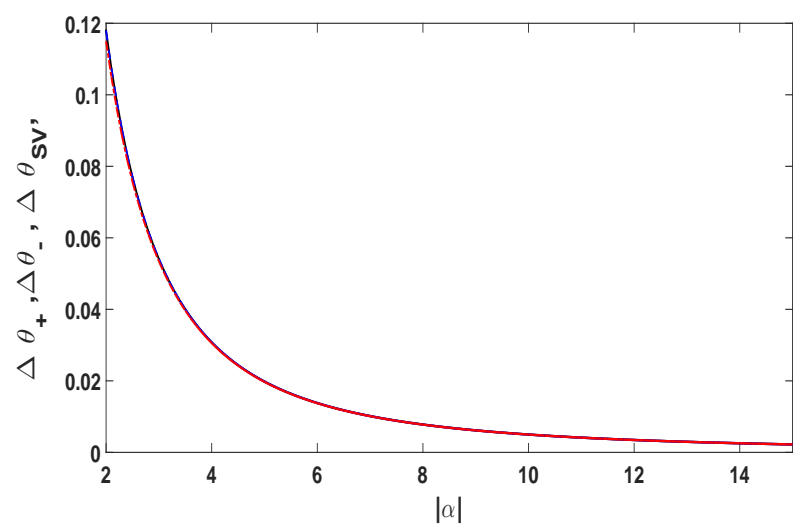

Fig. 6 Quantum Cramer-Rao bounds $\Delta \theta_{+}$(continuous), $\Delta \theta_{-}$(dashed) and $\Delta \theta_{\mathrm{SV}}$ (dot-dashed) are shown as a function of $|\alpha|$. Here we assume $\beta=|\alpha|, \theta=\pi / 2$. We choose $\alpha$ and $r$ such that $\left\langle\alpha,+\left|a_{1}^{\dagger} a_{1}\right| \alpha,+\right\rangle \approx\left\langle\alpha,-\left|a_{1}^{\dagger} a_{1}\right| \alpha,-\right\rangle \approx$ $\left\langle\xi\left|a_{1}^{\dagger} a_{1}\right| \xi\right\rangle$.

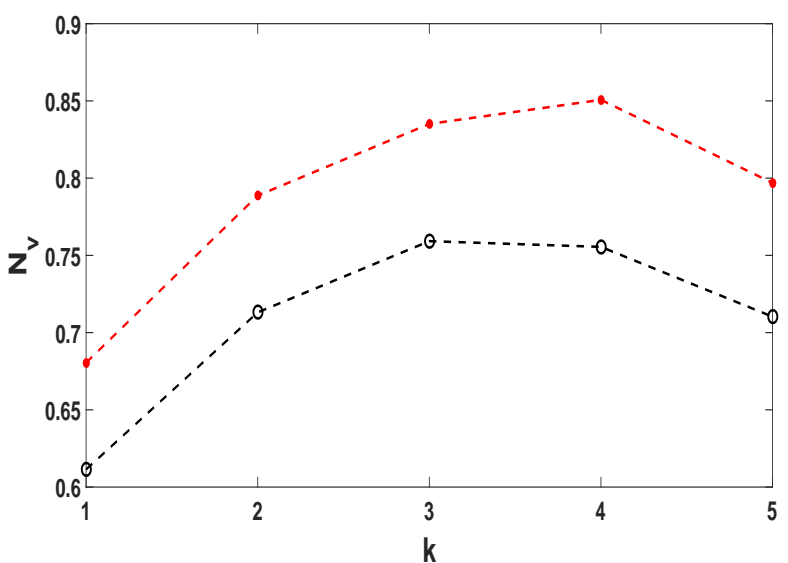

Fig. 7 Negativity of Wigner function for the states $|\psi(\alpha,\{m\})\rangle$ which achieve minimum $\Delta \theta_{\mathrm{M}}$. Here $\alpha=3 e^{i \pi / 2}($ circle) and $4 e^{i \pi / 2}$ (dot).

In this section, we discuss the role of nonclassicality on phase measurement using number state filtered coherent states. Nonclassicality of a state $|\phi\rangle$ is quantified in terms of the volume $\left(N_{v}\right)$ of the negative portion of Wigner function $W_{|\phi\rangle}(\chi)[30]$,

$$
N_{v}=\iint \frac{\left|W_{|\phi\rangle}(\chi)\right|-W_{|\phi\rangle}(\chi)}{2} d \chi^{\prime} d \chi^{\prime \prime}
$$

where $\chi^{\prime}$ and $\chi^{\prime \prime}$ are the real and imaginary part of $\chi$. For a given $|\phi\rangle$,

$$
W_{|\phi\rangle}(\chi)=\frac{2}{\pi} \sum_{n=0}^{\infty}(-1)^{n}|\langle\phi|D(\chi)| n\rangle|^{2},
$$

is the Wigner function for the state $|\phi\rangle[17,44]$. Here $D(\chi)=\exp \left(\chi a^{\dagger}-\chi^{*} a\right)$ is the displacement operator [17, 21]. 
Negativity of Wigner function for those states which achieve minimum $\Delta \theta_{\mathrm{M}}$ is shown as a function of $k$ in Fig. 7. Phase sensitivity increases as $k$ increases (refer Fig. 5) whereas the nonclassicality initially increases and then decreases as shown in Fig. 7. Hence, there is no guarantee that increasing nonclassicality will enhance phase sensitivity.

\section{Summary}

Coherent state and the vacuum as input states limit the phase sensitivity of Mach-Zehnder interferometer to the standard quantum limit which is inversely proportional to the square root of the mean number of photons in the input state. Surpassing this limit requires replacement of the vacuum by a nonclassical state. Phase sensitivity, quantified in terms of quantum Cramer-Rao bound, shows improvement beyond the standard quantum limit if number state filtered coherent states are used instead of the vacuum. Phase sensitivity increases further if many number states are removed from the coherent state. As an extension, the even and odd coherent states also classified as number state filtered coherent states. These states, with infinite number of states filtered, show significant improvement in phase sensitivity. For equal average number of photons, even and odd coherent states performance is as good as that of the squeezed vacuum as far as phase measurements are concerned. Though phase sensitivity increases with removing more number states from the coherent state, it is seen that nonclassicality does not increase monotonically. We will extend this work to study the effects of dissipation and decoherence on phase sensitivity to account for non-ideal mirrors and beam-splitters.

\section{Acknowledgement}

NM acknowledges Indian Institute of Technology Kanpur for postdoctoral fellowship.

\section{References}

1. Abramovici, A., Althouse, W.E., Drever, R.W.P., Gursel, Y., Kawamura, S., Raab, F.J., Shoemaker, D., Sievers, L., Spero, R.E., Thorne, K.S., Vogt, R.E., Weiss, R., Whitcomb, S.E., Zucker, M.E.: Ligo the laser interferometer gravitational-wave observatory. Science 256(5055), 325-333 (1992). DOI 10.1126/science.256.5055.325

2. Ataman, S., Preda, A., Ionicioiu, R.: Phase sensitivity of a mach-zehnder interferometer with single-intensity and difference-intensity detection. Phys. Rev. A 98, 043856 (2018). DOI 10.1103/ PhysRevA.98.043856. URL https://link.aps.org/doi/10.1103/PhysRevA.98.043856

3. Barnett, S., Fabre, C., Maitre, A.: Ultimate quantum limits for resolution of beam displacements. The European Physical Journal D - Atomic, Molecular, Optical and Plasma Physics 22(3), 513-519 (2003). DOI 10.1140/epjd/e2003-00003-3. URL https://doi.org/10.1140/epjd/e2003-00003-3

4. Birrittella, R., Mimih, J., Gerry, C.C.: Multiphoton quantum interference at a beam splitter and the approach to heisenberg-limited interferometry. Phys. Rev. A 86, 063828 (2012). DOI 10.1103/ PhysRevA.86.063828. URL https://link.aps.org/doi/10.1103/PhysRevA.86.063828

5. Bondurant, R.S., Shapiro, J.H.: Squeezed states in phase-sensing interferometers. Phys. Rev. D 30, 2548-2556 (1984). DOI 10.1103/PhysRevD.30.2548. URL https://link.aps.org/doi/10.1103/PhysRevD.30.2548

6. Boto, A.N., Kok, P., Abrams, D.S., Braunstein, S.L., Williams, C.P., Dowling, J.P.: Quantum interferometric optical lithography: Exploiting entanglement to beat the diffraction limit. Phys. Rev. Lett. 85, 2733-2736 (2000). DOI 10.1103/PhysRevLett.85.2733. URL https://link.aps.org/doi/10.1103/PhysRevLett.85.2733 
7. Braunstein, S.L., Caves, C.M.: Statistical distance and the geometry of quantum states. Phys. Rev. Lett. 72, 3439-3443 (1994). DOI 10.1103/PhysRevLett.72.3439. URL https://link.aps.org/doi/10.1103/PhysRevLett.72.3439

8. Buzek, V., Knight, P.L.: I quantum interference, superposition states of light, and nonclassical effects. Progress in Optics 34, 1 - 158 (1995). DOI https://doi.org/10.1016/S0079-6638(08) 70324-X. URL http://www.sciencedirect.com/science/article/pii/S007966380870324X

9. Campos, R.A., Gerry, C.C., Benmoussa, A.: Optical interferometry at the heisenberg limit with twin fock states and parity measurements. Phys. Rev. A 68, 023810 (2003). DOI 10.1103/PhysRevA.68.023810. URL https://link.aps.org/doi/10.1103/PhysRevA.68.023810

10. Caves, C.M.: Quantum-mechanical radiation-pressure fluctuations in an interferometer. Phys. Rev. Lett. 45, 75-79 (1980). DOI 10.1103/PhysRevLett.45.75. URL https://link.aps.org/doi/10.1103/PhysRevLett.45.75

11. Caves, C.M.: Quantum-mechanical noise in an interferometer. Phys. Rev. D 23, 1693-1708 (1981). DOI 10.1103/PhysRevD.23.1693. URL https://link.aps.org/doi/10.1103/PhysRevD.23.1693

12. Cramer, H.: Mathematical Methods of Statistics. Princeton University Press, Princeton, NJ (1946.)

13. Demkowicz-Dobrzanski, R., Jarzyna, M., Kolodynski, J.: Quantum limits in optical interferometry. Progress in Optics 60, 345-435 (2015)

14. Dodonov, V.V., Manko, V.I.: Theory of Nonclassical States of Light. Taylor and Francis group, LOndon (2003.)

15. Erol, V., Ozaydin, F., Altintas, A.A.: Analysis of entanglement measures and locc maximized quantum fisher information of general two qubit systems. Scientific Reports 4, 5422 (2014). URL https://doi.org/10.1038/srep05422

16. Escher, B.M., de Matos Filho, R.L., Davidovich, L.: General framework for estimating the ultimate precision limit in noisy quantum-enhanced metrology. Nature Physics 7, 406 (2011). URL https://doi.org/10.1038/nphys1958

17. Gerry, C., Knight, P.: Introductory Quantum Optics. Cambridge (England): Cambridge UP (2005.)

18. Gerry, C.C., Mimih, J.: Heisenberg-limited interferometry with pair coherent states and parity measurements. Phys. Rev. A 82, 013831 (2010). DOI 10.1103/PhysRevA.82.013831. URL https://link.aps.org/doi/10.1103/PhysRevA.82.013831

19. Giovannetti, V., Lloyd, S., Maccone, L.: Quantum metrology. Phys. Rev. Lett. 96, $010401 \quad$ (2006). DOI 10.1103/PhysRevLett.96.010401. URL https://link.aps.org/doi/10.1103/PhysRevLett.96.010401

20. Giovannetti, V., Lloyd, S., Maccone, L.: Advances in quantum metrology. Nature Photonics 5, 222 (2011). URL https://doi.org/10.1038/nphoton.2011.35

21. Glauber, R.J.: Coherent and incoherent states of the radiation field. Phys. Rev. 131, 2766-2788 (1963). DOI 10.1103/PhysRev.131.2766. URL https://link.aps.org/doi/10.1103/PhysRev.131.2766

22. Glauber, R.J.: The quantum theory of optical coherence. Phys. Rev. 130, 2529-2539 (1963). DOI 10.1103/PhysRev.130.2529. URL https://link.aps.org/doi/10.1103/PhysRev.130.2529

23. Hariharan, P.: Optical Interferometry. Elsevier (2003.)

24. Hayashi, M.: Phase estimation with photon number constraint. Progress in Informatics 8, $81-87$ (2011)

25. Helstrom, C.W.: Quantum detection and estimation theory. Journal of Statistical Physics 1(2), 231-252 (1969). DOI 10.1007/BF01007479

26. Jarzyna, M., Demkowicz-Dobrzanski, R.: Quantum interferometry with and without an external phase reference. Phys. Rev. A 85, 011801 (2012). DOI 10.1103/PhysRevA.85.011801. URL https://link.aps.org/doi/10.1103/PhysRevA.85.011801

27. Jonathan, P.D.: Quantum optical metrology the lowdown on high-n00n states. Contemporary Physics 49(2), 125-143 (2008). DOI 10.1080/00107510802091298 
28. Joo, J., Munro, W.J., Spiller, T.P.: Quantum metrology with entangled coherent states. Phys. Rev. Lett. 107, 083601 (2011). DOI 10.1103/PhysRevLett.107.083601. URL https://link.aps.org/doi/10.1103/PhysRevLett.107.083601

29. Joo, J., Park, K., Jeong, H., Munro, W.J., Nemoto, K., Spiller, T.P.: Quantum metrology for nonlinear phase shifts with entangled coherent states. Phys. Rev. A 86, 043828 (2012). DOI 10.1103/PhysRevA.86.043828. URL https://link.aps.org/doi/10.1103/PhysRevA.86.043828

30. Kenfack, A., Zyczkowski, K.: Negativity of the wigner function as an indicator of non-classicality. Journal of Optics B: Quantum and Semiclassical Optics 6(10), 396-404 (2004). DOI 10.1088/ 1464-4266/6/10/003

31. Kok, P., Lovett, B.W.: Optical Quantum Information Processing. Cambridge (2010.)

32. Lang, M.D., Caves, C.M.: Optimal quantum-enhanced interferometry using a laser power source. Phys. Rev. Lett. 111, 173601 (2013). DOI 10.1103/PhysRevLett.111.173601. URL https://link.aps.org/doi/10.1103/PhysRevLett.111.173601

33. Lang, M.D., Caves, C.M.: Optimal quantum-enhanced interferometry. Phys. Rev. A 90, 025802 (2014). DOI 10.1103/PhysRevA.90.025802. URL https://link.aps.org/doi/10.1103/PhysRevA.90.025802

34. Liu, J., Lu, X.M., Sun, Z., Wang, X.: Quantum multiparameter metrology with generalized entangled coherent state. Journal of Physics A: Mathematical and Theoretical 49(11), 115302 (2016). DOI 10.1088/1751-8113/49/11/115302

35. Lu, X.M., Wang, X., Sun, C.P.: Quantum fisher information flow and non-markovian processes of open systems. Phys. Rev. A 82, 042103 (2010). DOI 10.1103/PhysRevA.82.042103. URL https://link.aps.org/doi/10.1103/PhysRevA.82.042103

36. Ma, J., Huang, Y.x., Wang, X., Sun, C.P.: Quantum fisher information of the greenberger-hornezeilinger state in decoherence channels. Phys. Rev. A 84, 022302 (2011). DOI 10.1103/PhysRevA. 84.022302. URL https://link.aps.org/doi/10.1103/PhysRevA .84.022302

37. Meher, N., Sivakumar, S.: Number state filtered coherent states. Quantum Information Processing 17(9), 233 (2018). DOI 10.1007/s11128-018-1995-6. URL https://doi.org/10.1007/s11128-018-1995-6

38. Ozaydin, F., Altintas, A., Yesilyurt, C., Bugu, S., Erol, V.: Quantum fisher information of bipartitions of w states. Acta Physica Polonica A 127(4), 1233-1235 (2015). DOI http://dx.doi.org/ 10.12693/APhysPolA.127.1233

39. Ozaydin, F., Altintas, A.A.: Quantum metrology: Surpassing the shot-noise limit with dzyaloshinskii-moriya interaction. Scientific Reports 5, 16360 (2015). URL https://doi.org/10.1038/srep16360

40. Rao, C.R.: Linear Statistical Inference and its Applications. John Wiley and Sons (1973.)

41. Sudarshan, E.C.G.: Equivalence of semiclassical and quantum mechanical descriptions of statistical light beams. Phys. Rev. Lett. 10, 277-279 (1963). DOI 10.1103/PhysRevLett.10.277. URL https://link.aps.org/doi/10.1103/PhysRevLett.10.277

42. Tan, Q.S., Liao, J.Q., Wang, X., Nori, F.: Enhanced interferometry using squeezed thermal states and even or odd states. Phys. Rev. A 89, 053822 (2014). DOI 10.1103/PhysRevA.89.053822. URL https://link.aps.org/doi/10.1103/PhysRevA.89.053822

43. Wang, B.X., Tao, M.J., Ai, Q., Xin, T., Lambert, N., Ruan, D., Cheng, Y.C., Nori, F., Deng, F.G., Long, G.L.: Efficient quantum simulation of photosynthetic light harvesting. npj Quantum Information 4(1), 52 (2018). URL https://doi.org/10.1038/s41534-018-0102-2

44. Wigner, E.: On the quantum correction for thermodynamic equilibrium. Phys. Rev. 40, 749-759 (1932). DOI 10.1103/PhysRev.40.749. URL https://link.aps.org/doi/10.1103/PhysRev.40.749 